\title{
SAPER OSSERVARE la PUBblicita’ COME DOCUMENTO INTERCULTURALE
}

Elisabetta Pavan*

ABSTRACT: La pubblicità veicola la lingua, i gusti, la mentalità, i bisogni, le attitudini, i valori, in una parola la 'cultura' di un paese. In questo saggio vengono fornite delle indicazioni e degli strumenti di osservazione, oltre ad alcuni esempi di analisi, legati alla rappresentazione della cultura italiana in comunicati pubblicitari. Punto di partenza sarà guidare nella 'lettura' della pubblicità, chiarendo che essa è materiale autentico e che rappresenta lo spaccato di un Paese, riferibile al momento in cui il messaggio viene prodotto e trasmesso attraverso i vari mezzi di comunicazione di massa. Una pubblicità adeguatamente esaminata può fornire importanti indicazioni sul contesto socioculturale della cultura oggetto di studio e analisi. Imparare a 'leggere' la pubblicità vuol dire acquisire gli strumenti per poter seguire l'evoluzione della cultura rappresentata in spot televisivi, radiofonici, su internet, carta stampata e manifesti. Si tratta di un processo utile per gli apprendenti di una lingua straniera o seconda, in quanto aiuta a riflettere su aspetti verbali e non verbali della comunicazione, su valori, comportamenti, atteggiamenti, stili di vita, stereotipi; tuttavia le attività proposte sono valide anche per i madrelingua, in quanto aiutano a sviluppare consapevolezza culturale e interculturale e a conoscere e riconoscere la propria cultura e quella degli altri. Nel momento in cui ci si trova in una cultura che non è quella di appar- 
tenenza, attraverso la pubblicità, che va considerata un vero documento culturale, si possono intuire e scoprire valori che in quel momento sono accettati, e condivisi, dalla maggioranza della popolazione.

PAROLE CHIAVE: competenza interculturale, pubblicità, didattica della cultura, comunicazione interculturale, lingua straniera.

RESUMO: A publicidade veicula a língua, os gostos, a mentalidade, as necessidades, as atitudes, os valores, em resumo, 'a cultura' de um país. Neste ensaio são fornecidos indicações e instrumentos de observação, além de alguns exemplos de análises, relacionados à representação da cultura italiana em mensagens publicitárias. Ponto de partida será orientar na 'leitura' da publicidade, esclarecendo que ela constitui um material autêntico e que representa o recorte de um País, referente ao momento em que a mensagem é produzida e veiculada pelos diferentes meios de comunicação de massa. Uma publicidade analisada adequadamente pode dar indicações importantes sobre o contexto sociocultural da cultura que é objeto de estudo e análise. Aprender a ler a publicidade significa adquirir instrumentos que permitem acompanhar a evolução da cultura representada em anúncios televisivos, radiofônicos, na internet, em papel impresso e cartazes. Trata-se de um processo útil para o estudante de língua estrangeira ou de língua 2, porque o ajuda a refletir sobre aspectos verbais e não verbais da comunicação, sobre valores, comportamentos, atitudes, estilos de vida, estereótipos. Entretanto, as atividades propostas valem também para os falantes nativos porque os ajudam a desenvolver a consciência cultural e intercultural e a conhecer e reconhecer a sua própria cultura e aquela dos outros. No momento em que alguém se encontra numa cultura que não é a sua cultura nativa, por meio da publicidade, que deve ser considerada um verdadeiro documento cultural, pode intuir e descobrir valores que, naquele momento, são aceitos e compartilhados pela maioria da população.

PALAVRAS-CHAVE: competência intercultural, publicidade, didática da cultura, comunicação intercultural, língua estrangeira. 
ABSTRACT: Advertising / publicity, conveys, and is conveyed, by the language, tastes, mentality, needs, attitudes values, in other words, the culture of a group, or country. This paper offers suggestions / indications and tools for observing, and some examples of analyses of how Italian culture is represented in advertising / publicity communications. The point of departure is that of learning how to "read" advertising, this is authentic (real) material and it shows a picture of the country at the moment in which the message is produced and transmitted through the means of mass communication and the Media. Analysis of advertising and publicity can offer important information about the socio-cultural context, and about on-going changes, within the culture that is being analysed. As learning to "read" the messages behind advertising and publicity means acquiring the tools required to track changes, evolution, within a culture as revealed in radio and TV ads, on the internet, on poster adverts and in leaflets. This is a useful skill for those who are learning foreign languages as it helps them to reflect on the non-verbal, the cultural aspects of language, of communication, values, behaviour, lifestyles, stereotypes. It is also a useful skill for native speakers to acquire as it helps them to recognise and understand not only their own culture better, thus be able to analyse the cultural component in their own behavior and that of others, so enabling them to develop intercultural skills. When you find yourself in a culture that is not your own, it is possible to discover, to intuit, the values that are at that moment accepted and shared by, at least, the majority of the members of that new culture just by looking at the images, the values their advertisements and publicity transmit.

KEY-WORDS: Intercultural competence, advertising, culture teaching, intercultural communication, foreign language. 


\section{Introduzione}

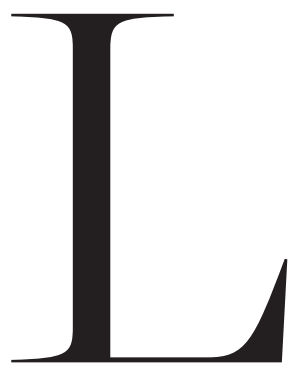

a pubblicità veicola la lingua, i gusti, la mentalità, i bisogni, le attitudini, i valori, in una parola la 'cultura' di un paese. Per questo è importante imparare a 'leggere' la pubblicità: si tratta di materiale autentico che rappresenta uno spaccato di un Paese, in questo saggio l'Italia, nel momento in cui il messaggio viene prodotto e trasmesso attraverso i vari mezzi di comunicazione di massa, e fornisce importanti indicazioni sul contesto socioculturale della cultura oggetto di studio e analisi.

Imparare a 'leggere' la pubblicità vuol dire acquisire gli strumenti per poter seguire l'evoluzione della cultura rappresentata in spot televisivi, radiofonici, su internet, carta stampata e manifesti, un processo utile per gli apprendenti di una lingua straniera o seconda, ma anche per i madrelingua, che porta a sviluppare consapevolezza culturale e interculturale, la prima riferita alla propria cultura (inculturazione), la seconda alla 'nuova' cultura (acculturazione).

In questo saggio vengono fornite delle indicazioni e degli strumenti di osservazione, oltre ad alcuni esempi di analisi legati alla rappresentazione della cultura 
italiana in comunicati pubblicitari, suggerendo che l'utilizzo di materiale pubblicitario autentico, sia a supporto dei libri di testo utilizzati, ma anche didattizzato in base alle specifiche necessità della classe e dell'insegnante, o utilizzato dal discente in maniera autonoma, possa aumentare la motivazione intrinseca e supportare l'acquisizione di una lingua e cultura, in un percorso di formazione che da guidata può diventare continua e autonoma. Utilizzeremo il termine (inter)culturale a sottolineare l'importanza della consapevolezza culturale e interculturale, distinte ma complementari.

\section{La pubblicità e il mercato}

La pubblicità influenza pensieri, sentimenti, la vita stessa; per questo il pubblico deve esserne consapevole e sviluppare una capacità critica che consenta di capirne il funzionamento. Essa offre immagini e valori, e il loro significato è veicolato da forme e strutture ben definite, affinché informazioni e concetti possano essere interiorizzati e il destinatario possa riconoscersi in essi.

Ogni giorno si vedono e si ascoltano innumerevoli messaggi pubblicitari: anche senza leggere il giornale, ascoltare la radio o guardare la TV, siamo sottoposti al bombardamento pubblicitario. Camminando a testa bassa per la strada non si può evitare la vetrina di un salumiere con esposti dei packaging (sono le confezioni sponsorizzate con il nome del produttore), per non parlare dei tabaccai, o dei supermercati, il vero cuore pulsante - o meglio, bancomat - dell'industria pubblicitaria. E se si resta a casa non si è salvi: ecco il volantinaggio nella cassetta della posta, e quando si accede a Internet subito di fronte all'utente si presentano ammiccanti e sgargianti i banner degli sponsor, che, per nulla casualmente, si rifanno a prodotti che sono strettamente collegabili a ricerche fatte in precedenza attraverso i vari motori o in siti di acquisto.

Viene così naturale considerare in maniera quasi rassegnata la pubblicità come inevitabile, qualcosa che ormai fa parte della nostra vita, ma sono in molti a non vederla in questo modo, e i pubblicitari sono tra questi. Essi, infatti, la considerano non uno dei meccanismi che si muovono all'interno del sistema, ma un carburante che consente al sistema di muoversi. La pubblicità diventa così uno dei fattori 
che può influenzare la nostra vita. Inoltre la pubblicità non si limita a 'vendere' dei prodotti: essa contribuisce a dare dei significati ai marchi produttori (es. Kraft. Cose buone dal mondo) e a modificare gli atteggiamenti del pubblico, attraverso, ad esempio, le campagne sociali.

La pubblicità è un messaggio intenzionale, deliberato, perché il suo scopo è quello di muovere all'azione potenziali consumatori. Quando il messaggio pubblicitario viene strutturato, esso ha sempre come riferimento il target, il segmento di pubblico che vuole colpire, non il mercato in generale. Ad esempio, tutti bevono Coca Cola, tuttavia ci sono pubblicità rivolte alle mamme che la comprano nei supermercati per i figli, altre rivolte ai ragazzi che la bevono al bar con gli amici, campagne per la stagione estiva e altre per la stagione invernale, e così via. Anche le banche in questo senso programmano pubblicità differenziate a seconda dei prodotti che vanno ad offrire (conto giovani, conto pensionati, fondi di investimento per casalinghe, fondi azionari o fondi pensione per manager rampanti, per poi scoprire, forse, che si tratta spesso dello stesso prodotto etichettato in maniera diversa).

Non occorre scendere in disquisizioni adatte più ad un marketing manager che ad un consumatore per intuire che la pubblicità fornisce e ripropone le idee e $\mathrm{i}$ valori che sono indispensabili all'esistenza di un determinato sistema economico. Obiettivo delle agenzie di comunicazione è che i prodotti siano comprati, usati e rimpiazzati, in un ciclo ininterrotto di consumo.

Alcune pubblicità possono risultare scontate, noiose, poco curate, $\mathrm{o}$ addirittura menzognere; altre, invece, si possono presentare come attraenti, ben impostate e addirittura divertenti. Tuttavia non va persa di vista la loro funzione primaria: una funzione prettamente economica (se si escludono le campagne con fini sociali o le pubblicità progresso) che si nasconde dietro alle scenette, agli effetti speciali, alle ambientazioni esotiche, ai testimonial.

La funzione principale della pubblicità è fornire al pubblico la conoscenza che sul mercato ci sono un certo numero di prodotti (informare) e dare così impulso al libero mercato, ma questo non è il solo ruolo ascrivibile ad essa. Negli anni, infatti, si è fatta sempre più invasiva e ha cercato di manipolare i valori sociali e le abitudini dei consumatori, tralasciando il ruolo di "informatrice" di prodotti e servizi (in alcuni paesi del sud Italia esiste ancora la figura del bardo, che 
nel giorno di mercato va di via in via intonando a gran voce una cantilena dove elenca i nomi delle attività commerciali, i prodotti e i prezzi del fruttivendolo, del salumiere, ecc.) e diventando "comunicatrice”. La funzione è quindi diventata quella di spingere al consumo, una risposta attiva come dimostrazione del coinvolgimento con la pubblicità stessa.

\section{La pubblicità come materiale glottodidattico}

La pubblicità è materiale autentico creato per madrelingua e con finalità diverse da quelle didattiche. È assodato che l'uso della pubblicità generi entusiasmo e metta lo studente in contatto con la lingua e la cultura quotidiane, svolgendo una funzione altamente motivante e rompendo la monotonia che talvolta si crea in classe (Mollica, 1979; Desideri, 1996; Lavinio, 1997; Caiazzo, Liotto, Morlicchio, 2005; Benucci, 2007).

Un'accurata selezione consentirà analisi sincroniche e diacroniche in una molteplicità di interrelazioni: la cultura italiana, ad esempio, può emergere da pubblicità create per un pubblico italiano in Italia, ma anche da pubblicità create all'estero per un pubblico eterogeneo e generalista o create all'estero per un pubblico di italiani o oriundi, ed essere analizzata sincronicamente per modello culturale, prodotto, mezzo di trasmissione, ecc. Una analisi diacronica consentirà, invece, uno studio in relazione ai cambiamenti nel tempo, dimostrand o contaminazioni e contatti che portano alla rappresentazione di 'spazi interculturali', intesi come aree di contatto tra culture e di adozione di modelli 'estranei’.

$\mathrm{Al}$ docente di lingua che vorrà utilizzare materiali autentici nelle sue lezioni suggeriamo di tener conto di vari aspetti didattici, quali il tipo di lingua, se LS (Lingua Straniera) o L2 (Lingua seconda), il livello linguistico dello studente, il tipo di abilità da sviluppare, l'età, la provenienza, la motivazione, la modalità di applicazione.

Per il docente la pubblicità è materiale grezzo da plasmare, didattizzare, in grado di collegare i bisogni linguistici degli studenti al sillabo programmato per un preciso livello linguistico, ed è uno stimolo per la riflessione su temi culturali, favorendo lo sviluppo di una consapevolezza (inter)culturale. Begotti (2008, p. 35) afferma che: 
il materiale ideale, per ottenere una relazione positiva con lo studente, deve proporre metodologie diversificate e stimoli multisensoriali al fine di sviluppare intelligenze, stili cognitivi e apprendimenti diversi, stimolando l'acquisizione linguistica a livello conscio e inconscio. (2008, p. 35).

Nella relazione positiva è insita la motivazione: con la didattizzazione della pubblicità, infatti, il docente può proporre alla classe materiale mirato, in grado sia di collegare i bisogni linguistici degli studenti al sillabo progettato, che di aumentare il coinvolgimento degli apprendenti, attivando il meccanismo di sfida cognitiva legato all'ipotesi dell' $\boldsymbol{i}+1$ (Krashen, 1982) e soddisfacendo i requisiti che vedono nel piacere l'energia motivante in grado di realizzare acquisizione (Titone, 1974; Balboni, 2002). Un coinvolgimento quasi totale, che con estrema facilità consente l'applicazione della rule of forgetting di Krashen. Inoltre, ad esclusione delle pubblicità radiofoniche, il messaggio commerciale presente sui vari mezzi sfrutta il supporto delle immagini facilitando, così, la comprensione. Baccin (2000, p. 45) sottolinea che:

per lo studente di italiano all'estero è importante confrontarsi con situazioni comunicative spontanee e naturali; prendere contatto con testi mirati sulla cultura e sulla civiltà italiane e confrontarsi con materiale autentico. Tutto ciò senza trascurare la competenza linguistica. Internet è una risorsa inesauribile di materiale autentico attualizzato, con testi di registri diversi di lingua, filmati ecc. L’uso didattico e ragionato del materiale autentico deve essere sfruttato al massimo dall'insegnante. (2000, p. 45).

Per una analisi semiologica del messaggio pubblicitario rimandiamo a Roland Barthes (1977) e all'analisi della pubblicità Pasta Panzani ${ }^{1}$ diffusa su carta stampata in Francia; nel saggio appaiono le definizioni di denotazione, connotazione e ancoraggio. Lo studioso fa corrispondere ad un primo livello visivo, quello della denotazione, il riconoscimento di determinate figure: pomodoro, funghi, barattolo, rete da pesca. Il secondo livello, quello della connotazione, si verifica nel momento in cui lo spettatore riconosce gli oggetti e li associa ad uno o più ulteriori significati, ricondicubili alla propria cultura o alla cultura di riferimento. Nella pubblicità analizzata da Barthes gli oggetti, grazie alla loro sostanza (peperone, pomodoro), richiamano 
alla mente del consumatore francese degli anni Sessanta la propria italianità; allo stesso modo i colori, più volte ripetuti, bianco (rete, formaggio), rosso (peperone, pomodoro, sfondo) e verde (ortaggi, confezioni) richiamano anch'essi all'italianità. Anche la struttura (layout) della pubblicità stessa ha un significato connotativo, in quanto richiama il genere pittorico della natura morta, un ulteriore riferimento all’italianità e all'arte pittorica, artisticità e valore estetico.

Un altro concetto riconducibile a Barthes, utile ai nostri scopi, è quello dell'ancoraggio, ovvero il rapporto fra testo verbale e visivo. Il testo verbale, infatti, può risultare fondamentale per la comprensione di una precisa denotazione dell'immagine, diversa da altre, concentrando l'attenzione dello spettatore su un aspetto preciso o un particolare dell'immagine stessa. L'ancoraggio svolge una funzione di controllo anche a livello connotativo, dando un indizio, segnalando una possibile traccia e portando così l'attenzione dello spettatore su una precisa interpretazione connotativa dell'immagine stessa. Il meccanismo dell'ancoraggio, una volta svelata la sua funzione e data la possibilità di riconoscere un preciso livello di interpretazione rispetto ad altri che possono essere ugualmente presenti, risulta particolarmente motivante per lo studente, che si sente gratificato dalla 'scoperta', come quando nella famosa immagine effetto ottico "la giovane e la vecchia” si riesce, dopo un po’ di sforzo, a riconoscere le due immagini, in maniera sequenziale e non contemporanea.

Il processo della percezione, in accordo con la teoria della psicologia della Gestalt, fornisce un'analisi della stessa, grazie alla quale è possibile definire il quadro di riferimento che fa 'vedere' il mondo prima che attualmente si veda con l'organo della vista, ovvero come le cose possano essere immaginate prima ancora che si abbia un contatto diretto con esse. Questa teoria ha evidenziato come colui che percepisce non lo faccia passivamente e sia portato ad organizzare gli stimoli che riceve in maniera coerente a seconda degli schemi che già possiede, creando una nuova percezione. Aggiungiamo che il processo di percezione e analisi della realtà avviene attraverso schemi che sono culturalmente connotati e che sono acquisti sin dalla più tenera età attraverso il processo di inculturazione. Saper osservare la pubblicità come documento interculturale può diventare uno strumento utilizzabile in un processo successivo, quello di acculturazione, in cui si riconoscono ed eventualmente adottano modelli culturali ascrivibili ad un'altra cultura. 
Grazie alla sua flessibilità e facilità di reperimento la pubblicità può essere considerata quindi un elemento utile non solo nella didattica di una lingua straniera, ma anche nella presentazione e acquisizione di modelli culturali, oltre che per sviluppare una abilità di analisi personale, il cui obiettivo è il raggiungimento di una personale consapevolezza (inter)culturale.

Sia nel caso di lingua straniera che nel caso di lingua seconda, il docente ha di fronte a sé rappresentanti di una società che è sempre più multiculturale, ed è per questo che deve ricordare che in classe ricopre la doppia funzione di singolo individuo e rappresentante della lingua e cultura target. Ecco quindi che la consapevolezza (inter)culturale va considerata un'abilità di base, parte integrante della più ampia competenza comunicativa. Il docente deve considerare questa abilità come qualcosa in continua evoluzione e non un obiettivo, un processo in cui osservazione, analisi e confronto sono i passi da affrontare per la conoscenza della nuova (ma anche della propria) cultura. Per una riflessione sulla comunicazione interculturale lato sensu e sulle relazioni che si possono sviluppare in classi multiculturali, oltre alle implicazioni legate all'insegnamento di aspetti culturali nell'ambito dell'insegnamento delle lingue straniere e seconde, rimandiamo a Pavan (2010).

\section{La pubblicità: un atto comunicativo interculturale}

La pubblicità può essere intesa come un atto comunicativo interculturale che avviene tra il pubblicitario e il pubblico in toto, in quanto persone che saranno esposte alla comunicazione pubblicitaria, e pubblico obiettivo (o target). Quest'ultimo sarà a sua volta distinto in target totale e core target, destinatario generico o mirato della comunicazione d'impresa, o sociale.

La prima distinzione si riferisce al pubblico a cui si rivolge il comunicato; ipotizziamo qui di seguito un esempio che non ha la pretesa di essere realistico né veritiero. La pubblicità di un detergente per il lavaggio a mano dei piatti, ad esempio, può essere vista da chiunque, adulti e bambini, persone che non avranno occasione o necessità di utilizzare, e/o acquistare il prodotto, perché non lavano i piatti o perché utilizzano la lavastoviglie, ma potrà essere vista anche da donne e uomini che lavano i piatti a mano, e potrà rivolgersi ad un pubblico maschile o femminile, 
appartenente ad una famiglia numerosa o mononucleare (proponendo, ad esempio, confezioni più piccole e con qualità più vicine alle preferenze di un pubblico femminile, come potrebbe essere una crema emolliente, o maschile, come l'inutilità del risciacquo). A seconda del target il messaggio verrà strutturato in modo da risultare più efficace per il pubblico di riferimento.

La seconda differenziazione, tra pubblicità d'impresa e sociale, può essere utile in quanto, mentre la comunicazione d'impresa ha l'obiettivo di muovere il destinatario all'azione, facendogli acquistare prodotti o servizi, la seconda intende modificare atteggiamenti di carattere 'sociale', ampliando l'area di consenso in relazione a tematiche di interesse per la collettività attraverso comunicati che vengono etichettati come pubblicità progresso, campagne sociali, propaganda, pubblicità no profit. I committenti possono essere pubblici, come, ad esempio, nella campagna di comunicazione sul "sostegno a distanza SAD”, trasmessa a cura del Dipartimento per il coordinamento amministrativo, in collaborazione con il Dipartimento per le politiche della famiglia e il Dipartimento per l'informazione e l'editoria ${ }^{2}$ e nella campagna "Sulla buona strada", o privati, come organizzazioni no profit. Rimandiamo al sito Osservatorio Campagne di Comunicazione Sociale ${ }^{4}$ per ulteriori campagne e materiali.

La comunicazione d'impresa consente un ampio e flessibile repertorio a cui attingere, in base agli obiettivi di analisi e divulgazione fissati dal docente o individuati dal discente nel caso di apprendimento autonomo, mentre la pubblicità sociale sarà particolarmente importante quando si vorranno presentare i comportamenti, gli atteggiamenti e i valori di una cultura.

Per l'analisi proposta in questo saggio suggeriamo di considerare i valori e gli atteggiamenti riferiti ad elementi quali classe sociale, benessere economico, sicurezza, tradizioni, identità nazionale, relazioni con gli stranieri e con le minoranze, politica, religione, correttezza politica. Per ulteriori strumenti di analisi suggeriamo le dimensioni individuate da Hosfstede (1991), quali individualismo e collettivismo, distanza dal potere, mascolinità e femminilità.

Sia il pubblicitario (inteso come gruppo creativo: copywriter, art director ecc.) che il potenziale pubblico appartengono ad una cultura che può essere la stessa, ma può anche essere diversa. Se consideriamo il fatto che ogni individuo appartiene a più co-culture e che le stesse sono intimamente legate alle diverse identità di ogni

2 http://www.governo.it/Presidenza/DICA/EVENTI/campagna_sad.html

3 http://www.mit.gov.it/mit/site.php?p=cm\&:o=vd\&id=1340

4 http://www.occs.it/ 
persona, e che ciascuno può legare ad ogni identità e co-cultura un diverso tipo di comunicazione, ogni scambio comunicativo può potenzialmente essere interculturale. Ad esempio una donna può essere italiana, ma anche madre, insegnante, giocatrice di tennis e ballare danze latino-americane: ecco definite una 'cultura' legata alla nazionalità e quattro co-culture che potranno influenzare la comunicazione a seconda che la donna si trovi con persone appartenenti allo stesso gruppo o con estranei.

Capire le differenze e individuare le analogie in un atto comunicativo che avviene coinvolgendo emittente e ricevente che appartengono a culture diverse è un importante punto di partenza per capire in che misura atteggiamenti, abitudini, comportamenti presenti nelle diverse culture possono determinare differenze o somiglianze. In seguito a questa consapevolezza verrà favorito il buon esito della comunicazione, in quanto la persona sarà in grado di utilizzare nuovi spunti di riflessione e strumenti di analisi.

La prospettiva da adottare in una analisi di questo tipo deve necessariamente essere variabile e tener conto del fatto che, all'interno di una stessa cultura, le differenze tra gli individui possono essere altrettante, se non addirittura superiori, a quelle che emergono tra gruppi di individui appartenenti a culture diverse. Grazie ai social network e ai mass media, ad esempio, adolescenti di paesi diversi condividono gusti e linguaggi che possono risultare estranei ai rispettivi genitori.

Per cultura intendiamo quel sistema di conoscenze, significati, azioni simboliche che uniscono un gruppo di persone che si riconoscono in essi. La cultura è invisibile, onnipresente e si impara: è un po' come una lingua, ogni bambino quando nasce può potenzialmente imparare qualsiasi lingua, ma, nella realtà, egli svilupperà le competenze in quella che sarà la sua madrelingua e inevitabilmente imparerà a riconoscere e riprodurre, in maniera automatica e il più delle volte inconsapevole, determinati suoni e non altri.

In questo saggio verrà volutamente tralasciato il messaggio "scontato" che può emergere da un comunicato pubblicitario ("lava più bianco", "il whisky per intenditori", "un'auto che è uno status symbol"); si cercherà altresì di analizzare la pubblicità per far emergere quanto di culturale, e non di commerciale, sia contenuto nel messaggio stesso, dando delle indicazioni che, al di là di essere analisi esaustive in relazione a comunicati specifici, possano essere utilizzate come indicazioni e stru- 
menti di riflessione applicabili ad altri messaggi pubblicitari che i lettori vorranno selezionare ed analizzare in base a preferenze o necessità.

\section{La pubblicità come comunicazione}

Decodificare un messaggio è quanto di più difficile possa esserci in un atto comunicativo: il ricevente, infatti, non potrà mai ricreare nella sua mente esattamente tutte le sfumature e i significati che l'emittente vorrebbe aver trasmesso; vanno inoltre considerati i distrattori, come le interferenze e i rumori di fondo. Il contesto è uno degli elementi da considerare nella decodificazione di un messaggio: in un ambiente di lavoro la comunicazione che avviene tra un dirigente e un impiegato vicino alla macchinetta del caffè molto probabilmente non avrà lo stesso tono di quella che ci sarà tra i due nell'ufficio del direttore.

Il contesto può essere definito anche background o scenario e, secondo l'analisi di Hymes, esso va distinto in setting e scene:

setting refers to the time and place of a speech and, in general, to the physical circumstances. Scene, which is distinct from setting, designates the "psychological setting”, or the cultural definition of an occasion as a certain type of scene. [...] In daily life the same persons in the same setting may redefine their interaction as a changed type of scene, say, from formal to informal, serious to festive, or the like. (1974, p. 55).

Bisognerà quindi tener conto del contesto fisico, reale, rappresentato nel comunicato pubblicitario, ma anche degli elementi culturali 'psicologici', i quali possono fornire importanti indicazioni in riferimento alla realizzazione dell'atto comunicativo.

Nella decodificazione di un messaggio pubblicitario, inoltre, si possono identificare un contesto reale e uno di fantasia. Il contesto reale è quello del ricevente nel momento in cui vede/sente la pubblicità: dal barbiere o dalla parrucchiera leggendo il giornale, mentre guida guardando le affissioni o ascoltando la radio, in salotto facendo zapping davanti alla TV; il contesto di fantasia si riferisce a quello che la 
pubblicità può evocare, che letteralmente 'fa sognare': le vacanze, un ambiente climatizzato, un invitante piatto di pastasciutta.

La pubblicità è un sistema di comunicazione in cui viene investito molto denaro, nonché ricerca, pianificazione, immaginazione e studio: tanto maggiore sarà la risposta in termini di "azione" da parte del target, tanto più la comunicazione avrà scambiato messaggi che hanno raggiunto l'obiettivo prefissato, e maggiore sarà il successo delle vendite legate al prodotto/servizio pubblicizzato.

L'uso della lingua varia a seconda del contesto, e considerare ogni pubblicità un atto comunicativo può aiutare a distinguere come, in una specifica cultura, certe forme di comunicazione e contenuti siano adeguati a determinate situazioni.

In questo saggio proponiamo un modello di analisi che abbiamo elaborato riferendoci al modello proposto da Hymes (1974) e a quello descritto nel Threshold Level (1990).

Gli elementi che abbiamo cercato di evidenziare, nella seppur rapida analisi di pubblicità italiane fatta in questo saggio, sono il luogo nello spazio, nel tempo e nella mente dei partecipanti, i partecipanti, l'avvenimento, la comunicazione, verbale e non verbale.

Ogni atto linguistico avviene in una situazione, e ogni situazione può essere scissa in vari elementi che la compongono, a partire dal luogo.

Iniziamo l'analisi dal luogo fisico in cui avviene la comunicazione, inteso come setting visibile; esso va considerato anche in maniera più ampia, valutando eventuali elementi che fanno da cornice al setting fisico, quali, ad esempio, l'istituzione o la regione geografica a cui esso appartiene o il fatto che sia uno spazio privato o pubblico.

Le istituzioni possono essere sociali, come ad esempio la famiglia o altri gruppi di appartenenza, o pubbliche, come organismi dello Stato, quali il servizio sanitario, la scuola, gli uffici pubblici, le associazioni del terzo settore, i partiti politici, o private, come imprese e industrie.

Lo spazio privato è quello che il parlante occupa in una pensione, in un hotel, in una pousada o in un bed and breakfast, ma anche in una casa o in un appartamento, propri, o di amici o parenti; lo spazio pubblico, invece, si riferisce a luoghi quali la piazza, i mezzi di trasporto, una stanza di ospedale, un ristorante, un centro sportivo, un luogo di culto, ambienti ove la presenza di persone sconosciute è prevedibile e non può essere messa in discussione. 
Il setting può essere analizzato anche in riferimento ad altre caratteristiche, quali, ad esempio, il fatto di essere uno spazio ricreativo, occupazionale, educativo.

Esiste poi un luogo nel tempo, ovvero il momento in cui avviene l'atto comunicativo, inteso come orario, giorno, stagione, periodo storico, e un luogo come elemento legato alla situazione psicologica dei partecipanti, che può riferirsi al modo in cui essi si sentono in quel momento o al ruolo che rappresentano, ad esempio, all'interno dell'istituzione che fa da cornice alla comunicazione, o all'interno della cultura di riferimento; l'ultimo elemento riferito al luogo in senso lato è l'avvenimento che fa da contesto alla situazione comunicativa, come, ad esempio, un evento sportivo, una festa in famiglia o di paese, un evento religioso, una vacanza, un viaggio in auto o in scooter, una pausa.

Queste ultime riflessioni introducono un altro elemento di analisi, le persone coinvolte nella situazione comunicativa: ogni persona rappresenta se stessa, il ruolo che ricopre in quella situazione, quello che ci si aspetta da lei e quello che rappresenta in quel momento.

Il contesto fisico include, oltre alle persone, oggetti e testi, scritti e orali, e le azioni compiute dai partecipanti.

Gli oggetti comunicano, ad esempio fornendoci precise indicazioni in qualità di status symbol, e i testi possono essere scritti, come sottotitoli, cartelli, titoli di libri o cartelloni presenti nel comunicato, o corrispondere al comunicato stesso, quando esso si realizza su carta stampata o internet, o orali, quando rappresentano il dialogo tra i partecipanti. Ricordiamo, inoltre, le varie competenze della comunicazione non verbale, come prossemica, cinesica, cronemica, oggettuale, tattile, olfattiva, e le loro caratteristiche, che possono variare da cultura a cultura.

Una pubblicità, sia essa statica come quelle che appaiono sulla carta stampata, $o$ in movimento, composta da audio e video o solo audio, può essere analizzata in tutte le sue componenti, portando alla luce elementi che si riferiscono alla competenza linguistico-comunicativa ma anche socio-culturale, e consentire una riflessione che porta alla consapevolezza culturale, o interculturale, a seconda dell'analisi e delle riflessioni condotte.

Una rapida conferma di quanto sin qui esposto si può avere quando non si capisce, o non si apprezza, completamente una pubblicità: in quel momento bisogna chiedersi se siamo noi che non ne abbiamo afferrato il senso o se effettivamente 
il comunicato si rivolge ad un target particolare e non abbiamo gli strumenti per decodificarla.

Portiamo l'esempio della campagna pubblicitaria per la televisione del prodotto IPhone di Apple trasmessa nel 2010: la campagna della compagnia telefonica $3^{5}$ si rivolge ad un target di giovani che ama viaggiare. La pubblicità, oltre a sfruttare la possibilità di avere immagini in movimento perché trasmessa in televisione, e di dimostrare quello che viene descritto a voce ("se vuoi una guida di Barcellona... se vuoi prendere confidenza con la metro... se vuoi mandare una cartolina a casa”), utilizza un tecnicismo che solo chi è introdotto nel mondo degli smartphone può capire: “Se vuoi mandare una cartolina a casa... anche per questo c’è un'app!", dove app è l'abbreviazione di applicazione, ovvero programma che consente lo svolgimento di una determinata funzione (come un software per il computer). La voce dello speaker rispecchia quella del target. Sicuramente una parte del pubblico che ha visto lo spot, trasmesso nei maggiori canali televisivi italiani, non sarà stata in grado di decodificare il significato di app, perché non usa, o non conosce, i cellulari di ultima generazione (smartphone).

La campagna della compagnia telefonica Vodafone ${ }^{6}$, invece, punta su un target più adulto, la voce narrante è sempre giovanile e il protagonista è una persona in grado di utilizzare uno smartphone, ma sono assenti i tecnicismi (non si parla di app) e sono invece presenti altri elementi, come la risoluzione delle immagini, la possibilità di leggere un quotidiano, usare i social media e filmare momenti della propria vita personale, caratteristiche più vicine ai bisogni e alle preferenze del target; allo scopo vien fatta vedere una bambina che mangia una fetta di anguria.

\section{Il lato culturale della pubblicità}

La pubblicità può essere definita atto comunicativo e, come le altre forme comunicative (il linguaggio, l'uso del corpo, gli oggetti, il silenzio...), essa ha degli aspetti che sfuggono alla programmazione razionale del messaggio stesso: colui o colei che produce o idea la pubblicità non sempre attribuisce un valore esplicito alle parole o alle immagini che la compongono. C’è sempre qualcosa che è dato per scontato, ed è proprio nel condiviso, nella certezza che il pubblico capirà, che la 
condivisione di determinati valori culturali gioca un ruolo fondamentale. In Italia, ad esempio, la tazzina del caffè, o del cappuccino, o il tazzone (mug inglese) sul tavolo della cucina quasi certamente indicano colazione, mentre un paio di uova al tegamino con pancetta possono essere il segnale che si tratta di una cena veloce; le stesse immagini in Inghilterra potrebbero essere decodificate al contrario, per questo vanno considerati sia setting che scene.

Due amici seduti al tavolino di un bar che bevono un cappuccino stanno presumibilmente chiacchierando su temi personali e si stanno prendendo una pausa di mattina; ma se uno sguardo più attenti ci fa riconoscere un setting che non è un bar italiano ma una panetteria brasiliana, il tempo può slittare e potrebbero essere due amici, che stanno chiacchierando su temi personali, e si stanno prendendo una pausa di mattina $\mathrm{o}$, con tutta probabilità, di pomeriggio.

Il pubblicitario utilizza linguaggio, immagini, idee e valori caratteristici della sua cultura e li usa per comporre un messaggio pubblicitario che sarà decodificato all'interno di una cultura, o meglio, all'interno della mente di un componente una determinata cultura. A volte le immagini sono uno spaccato della vita reale degli italiani, altre volte sono stucchevoli composizioni di "famiglie felici" (come alcune pubblicità del marchio Mulino Bianco di Barilla), immagini che hanno ben poco a che fare con la realtà quotidiana.

Il consumatore non è una figura indipendente che autonomamente formula le sue decisioni d'acquisto: il consumatore è, in senso lato, il prodotto della comunicazione in una determinata cultura. Ricordiamo, allo scopo, l'affermazione di E.T. Hall (1976, p. 186): “culture is communication, and communication is culture”. In questo senso il consumatore va considerato un risultato: non è colui che muove le leve e influenza la pubblicità ma un effetto, ovvero si muove come ha imparato a fare nella sua cultura e la pubblicità lo stimola a farlo secondo i suoi - della pubblicità fini, toccando precise leve culturali.

Una comunicazione commerciale sarà tanto più efficace quanto più saranno rilevanti al suo interno i riferimenti alla cultura più popolare, nel senso di in auge, in quel momento per il core target. Il pubblico nel decodificare il messaggio, a livello conscio o subconscio, userà tutti i contesti, codici linguistici, miti e metafore a sua disposizione per effettuare le connessioni culturali che potranno collegare il consumatore al prodotto/servizio o al mercato. È quindi importante che il prodotto 
sia presentato nel cuore della cultura più popolare del momento per poter colpire un numero maggiore di consumatori. Si pensi, ad esempio, alle pubblicità legate ai gestori della telefonia mobile in Italia: gli spot cambiano di frequente, a volte ci sono delle miniserie in cui i soggetti si ripetono in vari episodi, ma quello che conta, ai fini della nostra analisi, è che i richiami alla realtà del momento siano davvero il cuore dello spot e ci siano collegamenti con la realtà del momento. Due campagne pubblicitarie di telefonia mobile trasmesse prima della stagione estiva affrontano in maniera diversa l'elemento vacanze estive per studenti adolescenti. La campagna pubblicitaria di TIM presenta due fidanzatini che sono prima all'uscita da scuola fine anno scolastico - e poi in vacanza: tavolino, ombrellone, mare. Omnitel invece ha optato per un setting prima curiosamente da Indiana Jones e poi improvvisamente casalingo, dove il ragazzino gioca con la sua Playstation.

\section{Analisi (inter)culturale della pubblicità}

Nel Common European Framework o Quadro Comune Europeo di riferimento (QCER, Council of Europe, 2001) si afferma che la conoscenza della società e della cultura della comunità in cui si parla una determinata lingua è uno degli aspetti della conoscenza del mondo. Possiamo affermare che questa conoscenza è spontanea in riferimento alla cultura di appartenenza, talvolta non strutturata e viziata da stereotipi per le altre culture. Nel QCER vengono elencati degli elementi, a loro volta declinati in vari componenti, che possono essere utili indicatori per una analisi (inter)culturale. Ci rifacciamo all'elenco presente nel testo originale, adattandolo all'obiettivo del saggio.

1. La vita di tutti i giorni: cibi e bevande, orari dei pasti, buone maniere a tavola; ferie; consuetudini e orari sul posto di lavoro; attività nel tempo libero (hobby, sport, letture, media);

2. Le condizioni di vita: tenore di vita e varianti legate ad elementi regionali, etnici e sociali; abitazione; previdenza sociale.

3. Relazioni interpersonali (compresi rapporti di potere e solidarietà) in funzione di: strutturazione di classi sociali e rapporti al loro interno; rapporti tra i sessi (genere e intimità); struttura e rapporti nella famiglia; rapporti tra generazioni; 
rapporti sul posto di lavoro; rapporti tra cittadini e polizia, pubblici ufficiali ecc.; rapporti tra diversi gruppi etnici e comunità; rapporti tra gruppi politici e religiosi.

4. Valori, convinzioni e atteggiamenti, in relazione a elementi quali: classi sociali; categorie professionali (docenti, dirigenti, personale di servizi pubblici, lavoratori specializzati, manovalanza); patrimonio (guadagni o beni acquisiti per eredità); culture regionali; sicurezza; istituzioni; tradizioni e cambiamenti sociali; storia, in riferimento ad avvenimenti storici e personaggi dal valore simbolico; minoranze etniche e religiose; identità nazionale; paesi e popoli stranieri; politica; arti, quali musica, arti visive, letteratura, teatro, canzoni popolari; religione; umorismo.

5. Il linguaggio del corpo, nei suoi elementi di azioni concrete che accompagnano le attività linguistiche ma anche negli aspetti relativi alla comunicazione non verbale e ai significati simbolici da essa veicolati, che possono essere diversi da cultura a cultura. Tra questi ricordiamo gesti, espressioni del volto, postura, contatto oculare, contatto fisico, prossemica; l'uso di onomatopee ('sst!' per imporre il silenzio) e i tratti prosodici (lunghezza, tono, accento, volume, tipo di voce). La conoscenza delle convenzioni che governano questi elementi sono parte della competenza socioculturale.

6. Le convenzioni sociali, ad esempio in riferimento a dare e ricevere ospitalità: puntualità; regali; abbigliamento; rinfreschi, bevande e pranzi; convenzioni e tabù nel comportamento e nella conversazione; durata della visita; il modo di accomiatarsi.

7. I comportamenti rituali in aree quali pratiche e riti religiosi; nascita, matrimonio, morte; comportamento di ascoltatori e spettatori di spettacoli e cerimonie; celebrazioni, festività, balli, discoteche.

Nel QCER (Council of Europe, 2001) si dichiara che alla base della consapevolezza interculturale stanno la conoscenza, la consapevolezza e la comprensione del rapporto (somiglianze e differenze) esistenti tra il mondo di origine e il mondo della comunità di cui si impara la lingua, e, aggiungiamo noi, la cultura, compresa la visione che ogni comunità ha dell'altra, gli stereotipi e gli autostereotipi nei due sensi.

Proponiamo in qualità di esempio di analisi cross-culturale, una analisi sincronica della campagna pubblicitaria televisiva di un detergente in polvere per lavatrice, il Dixan, nelle sue varianti per il mercato italiano e per il mercato spagnolo. 
A differenza di altri detersivi, il Dixan mantiene lo stesso nome nei due mercati, europei e mediterranei. Si potrebbe pensare che lo stesso prodotto, con lo stesso nome, in due paesi mediterranei come la Spagna e l'Italia mantenesse la stessa strategia comunicativa: e invece non è così. Nella pubblicità spagnola il setting è un giardino con alberi, piscina; una donna sui quarant'anni in costume da bagno e con capelli bagnati si avvicina al lettino sul bordo della piscina e con disappunto espresso da un "Oh no, otra mancha de bronzeador" scopre una macchia sull'asciugamano. Dalla piscina emerge il Signor Dixan (l'attore italiano Andrea Giordana) che conduce la signora in un viaggio all'interno del tessuto. Le illustra in che modo le macchie si legano alle fibre e come Dixan riesce a sconfiggere anche le macchie più ostiche già a bassa temperatura grazie alle sue perle attive. Durante il viaggio la signora è abbagliata dal biancore del tessuto e l'attore le offre un paio di occhiali da sole. I due escono dai tessuti, si avvicinano all'asciugamano che nel frattempo è miracolosamente diventato bianco e brillante. Immagine della confezione del prodotto e pay off.

Il setting della pubblicità realizzata per la diffusione nei media italiani è un giardino con alberi, dove ci sono corde da bucato tese con lenzuola appese. Una donna sui quarant'anni è alle prese con la biancheria e scopre delle macchie che non prevedeva. Il setting è un ampio prato verde dove ci sono delle corde da bucato tese e delle lenzuola stese. Appare il Signor Dixan (l'attore italiano Andrea Giordana) che conduce la signora in un viaggio all'interno del tessuto. Le illustra in che modo le macchie si legano alle fibre e come Dixan riesce a sconfiggere anche le macchie più ostiche già a bassa temperatura grazie alle sue perle attive. Durante il viaggio la signora è abbagliata dal biancore del tessuto e l'attore le offre un paio di occhiali da sole. Escono dai tessuti, si avvicinano ai panni che sono miracolosamente bianchi e brillanti. Immagine della confezione del prodotto e pay off (slogan finale in cui viene descritto il prodotto).

Il setting dei due spot potrebbe essere lo stesso: il giardino di una villetta, ampio prato, alberi, il tutto ben curato, a indicare un reddito medio alto, ma diversi sono gli stili di vita delle due donne.

Nello spot spagnolo si vedono la piscina e un muretto, probabilmente di fianco c'è un'altra abitazione; nello spot italiano non si vede la piscina ma sussiste la recinzione, potrebbe trattarsi dello stesso giardino ripreso da una differente ango- 
lazione. Nelle due pubblicità possiamo leggere i valori legati alla figura della donna nei due paesi. Al di là dello stereotipo mamma, in Spagna la donna si è guadagnata uno spazio suo che le consente di essere rappresentata sola sul bordo di una piscina e di essere colei che macchia di abbronzante l'asciugamano (è artefice delle sue macchie). In Italia, invece, è alle prese con le faccende domestiche (il bucato da stendere nell'ampio prato all'inglese, una maglietta macchiata di verde che appartiene al figlio calciatore o una tovaglia macchiata di vino rosso) e lava le macchie che qualcun altro ha fatto. Questa figura di donna rispecchia il valore ancora molto maschile della nostra società, dove la donna continua a essere vista come moglie/ mamma in uno spazio che è quello della famiglia, pronta a risolvere eventuali problemi che altri hanno causato.

\section{Esempi di analisi}

Uno sguardo attento a come la figura femminile viene rappresentata nelle pubblicità può dare delle indicazioni al ruolo che viene attribuito alla donna in una particolare cultura.

Dall'analisi, anche sommaria, di un campione di comunicati pubblicitari italiani emerge un'idea di donna legata alla famiglia, pronta a risolvere qualsiasi tipo di problema, dalle macchie , alla stipsis ${ }^{\text {s }}$ alla perdita di peso, alla caduta dei capelli, alla cucina sana e naturale $o$ all'assistente che risponde alle domande dei consumatori'; raramente la donna viene rappresentata vicino ad un computer o sul posto di lavoro. Per quanto riguarda prodotti dimagranti o di bellezza, di solito il testimonial è donna ${ }^{10}$; nello stesso sito è possibile vedere anche lo spot trasmesso in televisione per pubblicizzare lo stick da bere: nei sei quadri presentati, uno è dedicato alla descrizione del prodotto, in cinque è presente un testimonial donna, in uno un uomo.

La figura femminile viene sporadicamente associata al prodotto automobile, di solito è seduta di fianco al guidatore, lo stesso vale per il prodotto scooter" ${ }^{11}$. Talvolta la figura della donna è protagonista nella comunicazione pubblicitaria di questi prodotti: nella campagna italiana rappresenta un sex symbol, quasi una chiosa del motto 'donne e motori' (ad esempio nella versione italiana della pubblicità di Alfa Romeo Giulietta, il cui slogan "Noi siamo fatti della stessa materia di cui sono

\footnotetext{
7 http://comunicazionedigenere.wordpress.com/tag/dixan/

8 http://nonpalline.blogspot.it/2010/02/intestini-ribelli.html

9 http://www.nutrifree.it/media/img/repository/521_istituzionale-aprile2010.jpg

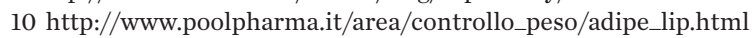

11 http://www.sphitalia.com/portfolio/lml-3/\#prettyPhoto, http://www.motorionline.com/2009/11/11/piaggio-

liberty-e-liberty-elle-gamma-2010/
} 
fatti i sogni” si rifà a Shakespeare²), mentre nella versione spagnola la testimonial mostra una profonda scollatura e sorride ${ }^{13}$. La giovane donna è talvolta tentatrice: assente un ipotetico compagno, invita l'amico a casa a bere un limoncello, classico liquore a base di limoni originario della costiera amalfitana.

Analizzando le pubblicità si possono trarre indicazioni sui rapporti di coppia: in riferimento alla fascia di età che va dai venti ai trent'anni emerge un rapporto piuttosto instabile, tuttavia negli spot in cui viene rappresentata l'immagine della famiglia con figli la donna non manca mai; la rappresentazione della famiglia monoparentale è quasi inesistente. A grandi linee si può dire che la donna, in Italia, è ancora una figura di supporto, moglie e mamma, detiene i valori legati alla femminilità (prodotti di bellezza, compagna dell'uomo) e maternità (prodotti alimentari, per l'igiene di casa e famiglia, vacanze con marito e bambini). Nell'ambito lavorativo è difficile per un uomo accettare che una donna gli sia superiore in gerarchia, $\mathrm{e}$ questo si può dedurre dalla quasi totale assenza di donne manager negli spot analizzati; se la donna manager viene rappresentata, di solito indossa il tailleur con la gonna, non con i pantaloni!

È interessante notare come il prodotto scooter sia raramente associato all'immagine del ragazzino, anzi, soprattutto dall'entrata in vigore della legge che rende obbligatorio l'uso del casco per tutti i ciclomotori, lo scooter spesso viene cavalcato da un ex-yuppi con cravatta che sventola di lato, che indossa un completo scuro, tiene valigetta tra le gambe o sul portapacchi e sta usando il cellulare con l'auricolare ${ }^{14}$. Proponiamo una pubblicità televisiva in cui si possono riconoscere il non rispetto per le regole in fatto di parcheggio (lo scooter è sul marciapiede), il calcio sport nazionale (la radiocronaca di calcio come colonna sonora dello spot) e la vigilessa che non fa la contravvenzione ${ }^{15}$.

L'automobile è un prodotto che richiederebbe una analisi piuttosto complessa, in quanto le campagne dello stato per incentivare la rottamazione e il passaggio a veicoli meno inquinanti ha favorito un succedersi di campagne pubblicitarie più tattiche che strategiche. Infatti sono molte le campagne che puntano sugli sconti e sulle facilitazioni d'acquisto, un po' meno quelle che si focalizzano sul prodotto. Segnaliamo una campagna che ha sfruttato molto il mezzo radiofonico, un jingle che è diventato un tormentone ( $\mathrm{Ga}$ el SUV), anche per la sua caratteristica di essere cantata da un DJ rapper milanese le cui canzoni parlano di rispetto per gli altri e

\footnotetext{
12 http://www.rubric.it/meltingpot/romeo-giulietta-e-la-thurm/ 13 https://encrypted-tbno.gstatic.com/ images?q=tbn:ANd9GcSb0LYzULlpShmBMqtWMlRBesgW79HycgHLlU6wAa8RvrkoYmttHw 14 http://www.omnimoto.it/magazine/5030/kymco-people-gti-300-e-125, http://www.omnimoto.it/foto/ popup/53163/kymco-people-gti-ipad-iphone

15 http://www.youtube.com/watch?v=xRl87VFjqhg
} 
dell'amore per la sua Milano, città bellissima ma assediata dal traffico, che è stata declinata nelle varianti napoletana e romana ${ }^{16}$. In questa pubblicità, oltre alle varianti linguistiche regionali, va notato il contenuto in riferimento a traffico e abitudini dei giovani nel traffico, ma anche la presenza nel gruppo di un elemento dai tratti somatici non italiani e il rispetto per le regole stradali.

Il mezzo aereo sfrutta soprattutto campagne legate alle offerte tariffarie; la ferrovia punta su target diversi a seconda delle facilitazioni proposte. L'utente dell'aereo è quasi sempre un elegante uomo d'affari, a volte appare solo la hostess; per la ferrovia i testimonial sono differenziati: nonni, giovani amiche in vacanza, uomini d’affari, mai i pendolari.

La quantità di comunicati di auto e moto è nettamente superiore a quella di aerei e treni, che sfruttano di più l'internet. Facile dedurre che in questo momento, in Italia, il mezzo di trasporto che "tira" è su gomma, e l’immagine tipo del conduttore di mezzi su gomma è maschile, tra i trenta e i quarant'anni, vestito elegantemente, solo o accompagnato da una bella donna, su auto di media e grossa cilindrata, con la famiglia se si tratta di monovolume o station wagon. Le pubblicità che ritraggono giovani o gruppi di amici sono una esigua minoranza; tra queste segnaliamo, oltre al già citato SUV, lo spot della Fiat per il modello Doblò ${ }^{17}$, dove protagonisti sono degli uomini apparentemente giamaicani, che in un setting tropicale e allegro incontrano le loro compagne, di etnia caucasica e incinta, e si allontanano felici a bordo del loro mezzo. Lo straniero di origine africana nelle pubblicità viene rappresentato in maniera completamente diversa da quella che si può trovare negli articoli di cronaca della carta stampata.

La donna al volante non appare quasi mai se non su modelli tipo utilitaria; non sono emerse pubblicità in cui la donna sia al volante e l'uomo al suo fianco (il contrario invece è molto frequente).

La famiglia degli spot e degli annunci è sempre una famiglia felice. A cominciare dalla colazione del mattino: luci dorate, l'aroma del caffè ‘vero' o quello di orzo (siamo in Italia, il caffè si fa con la caffettiera), i biscotti o brioche (cornetti) o merendine calde da forno ${ }^{18}$.

Il caffè si beve anche la sera purché decaffeinato, e il caffè della macchina si chiama espresso anche in Italia, ma solo quando si fa a casa ${ }^{19}$, quello del bar è, infatti, unico e speciale, ma solo un caffè ${ }^{20}$.

$16 \mathrm{Ga}$ El Suv http://www.youtube.com/watch?v=j_DzmKztge8, http://www.youtube.com/watch?v=2yuE-X3Vl9Y, http://www.youtube.com/watch?v=Hwlea33dkxk, integrale http://www.youtube.com/watch?v=5VM97nmollc\&NR $=1 \&$ feature $=$ endscreen

$17 \mathrm{http}: / /$ www.youtube.com/watch?v=oqzDjqf_Hec

18 http://www.youtube.com/watch?v=kLtlabWAHPE, http://www.youtube.com/watch?v=PhNM88v9z64, http:// www.youtube.com/watch?v=fF-whqelilw $19 \mathrm{http}: / /$ www.youtube.com/watch?v=6HD-u96jDrA

20 http://www.youtube.com/watch?v=f5VJYb3R-9U 
La famiglia viene prevalentemente usata per beni di consumo: la vacanza, la colazione, alimentari in genere (latte, yogurt, formaggio...), più raramente per beni durevoli quali l'abitazione. Per quanto riguarda la casa, la famiglia viene usata moltissimo nelle pubblicità delle banche, soprattutto per il prodotto mutuo fondiario.

La famiglia italiana 'ideale' è composta da madre, padre, figlio maschio e figlia femmina in età scuola elementare. Quando si passa all'età adolescenziale la figlia viene spesso rappresentata insieme alla madre e il figlio con il padre: segnale che la comunicazione tra i sessi è difficile, non solo per una questione generazionale ma soprattutto per motivi culturali legati alla differenziazione dei ruoli tra i sessi.

La figura del nonno o della nonna è legata alla saggezza dell'età: la nonna dai capelli turchini sa sempre come smacchiare senza strappare, il nonno è un po' più pasticcione e a volte anche diseducativo (nello spot Philadelphia induce il nipotino alla menzogna); quando invece si tratta della suocera quest'ultima è sempre in competizione con la nuora, quasi a combattere una ipotetica battaglia per la riconquista del figlio, tipica scenetta alla base di molte pubblicità di detersivi per il bucato e per l’igiene della casa.

Il mondo degli affari è un mondo prettamente maschile. Una analisi relativa sia al commercio che agli impieghi di concetto fa emergere una predominante presenza dell'uomo. Il consulente finanziario è di solito un interessante ragazzotto sui trent'anni; il salumiere è sempre un uomo sui cinquanta.

Tuttavia le donne al lavoro non mancano: commesse, impiegate o possibili promoter che in minigonna fanno svolazzare le gambe finalmente leggere dopo aver assunto delle pastiglie specifiche contro la pesantezza agli arti inferiori, quasi che gli uomini non soffrissero di disturbi alla circolazione. Anche l'esperto in macchie che si materializza nei due spot Dixan analizzati all'inizio è un uomo, come se le materie tecnico-scientifiche fossero appannaggio del sesso forte. Quando la donna manager viene rappresentata c'è sempre un sottile velo di ironia, quasi che la scalata al successo fosse una concessione e non una conquista. 


\section{Conclusioni}

La nostra società è un mosaico culturale, e persone di culture differenti possono intendere in maniera a volte impercettibilmente diversa il sistema di valori, il modo di ragionare, la maniera di comunicare, i comportamenti e le usanze.

Saper osservare la pubblicità può essere un ottimo mezzo per riuscire a individuare determinati valori culturali, conoscere e riconoscere la propria cultura e quella degli altri. Nel momento in cui ci si trova in una cultura che non è quella di appartenenza, attraverso la pubblicità si possono intuire e scoprire valori che in quel momento sono accettati, e condivisi, dalla maggioranza della popolazione: per definizione un pubblicitario cercherà sempre di colpire il target più ampio possibile, quindi nella costruzione dello spot saranno molteplici i riferimenti alle necessità e consuetudini del momento. La pubblicità può anche aiutare a restare in contatto con la realtà del proprio paese quando si è lontani e comunque, al di là delle informazioni relative al prodotto, in ogni comunicato si possono leggere valori, stili di vita, abitudini e stereotipi, proprio come in un documento culturale.

Grazie alla sua flessibilità e facilità di reperimento la pubblicità può essere considerata un elemento utile non solo nella didattica di una lingua straniera, ma anche nella presentazione e acquisizione di modelli culturali, oltre che per sviluppare una abilità di analisi personale, il cui obiettivo è il raggiungimento di una personale consapevolezza (inter)culturale.

Saper osservare la pubblicità come documento interculturale, utilizzando strumenti e strategie suggeriti in questo saggio, rientra negli obiettivi promossi da un lifelong learning, un apprendimento continuo ma, soprattutto, autonomo.

\section{Bibliografia}

BACCIN, P.G. Giochi e strategie ludiche per l'insegnamento dell'italiano, in Anais do VII Congresso Nacional de professores de Italiano e II Congresso Internacional de Estudos italianos, Belo Horizonte, 2000, v. I.

BALBONI, P.E. Le sfide di Babele. Insegnare le lingue nelle società complesse, UTET Libreria, Torino, 2002.

BARTHES, R. Rhetoric of the Image, in Image, Music, Text, S. Heath, 1977, ( a cura di e traduzione), Hill and Wang, New York, 1964. 
BEGOTTI, P. Dalla pubblicità alle canzoni: didattizzare materiale autentico per insegnare l’italiano a stranieri, in ITALS Didattica e linguistica dell'italiano come lingua straniera, VI, 17, 2008, pp. 33-50.

BENUCCI, A. Lingue straniere e pubblicità: pubblicità televisiva e insegnamento dell italiano, in M. Cardona (a cura di) Vedere per capire e parlare, UTET Università, Torino, 2007.

CAIAZZO L., LIOTTO M. R., MORLICCHIO E. La pubblicità anima la didattica, In Lavinio C. (a cura di) Educazione linguistica e educazione letteraria. Intersezioni e interazioni, Franco Angeli, Milano, 2005.

COUNCIL of Europe. A common European framework of reference for languages: Learning, teaching, assessment. Council of Europe, Strasbourg, 2001.

DESIDERI, P. (a cura di). La pubblicità tra lingua e icona, Humana Editrice, Ancona, 1996.

HALL, E. T. The Silent Language. Doubleday, New York, 1959.

HOFSTEDE, G. Cultures and organizations: Software of the mind, McGraw-Hill, New York, 1991.

HYMES, D. Foundations in Sociolinguistics: An Ethnographic Approach, University of Pennsylvania Press, Philadelphia, 1974.

KRASHEN S. Principles and Practice in Second Language Acquisition, Prentice-Hall, New York, 1982.

LAVINIO, C. La pubblicità nella didattica, in Crescere con la pubblicità. Didattica e comunicazione pubblicitaria, in G.L. Quartu (a cura di), Artigianarte, Cagliari, 1997.

MOLLICA, A. A Tiger in Your Tank: Advertisements in the Language Classroom, in «The Canadian Modern Language Review/La Revue Canadienne des Langues Vivantes», 35,4, 1979, pp. 691-473.

PAVAN, E. La dimensione interculturale nella comunicazione: una questione di consapevolezza, in Le lingue dell'educazione in un mondo senza frontiere, in MEZZADRI M. (a cura di), Guerra Edizioni, Perugia, 2010.

TITONE, R. Insegnare oggi le lingue seconde, Società Editrice Internazionale, Torino, 1979. 